\title{
ATF4 regulated by MYC has an important function in anoikis resistance in human osteosarcoma cells
}

\author{
HAO MO*, JIAN GUAN ${ }^{*}$, LIGEN MO, JULIANG HE, ZHENJIE WU, XIANG LIN, \\ BIN LIU and ZHENCHAO YUAN
}

\begin{abstract}
Department of Bone and Soft Tissue Neurosurgery, Affiliated Tumor Hospital of Guangxi Medical University, Nanning, Guangxi 530021, P.R. China
\end{abstract}

Received March 24, 2016; Accepted May 2, 2017

DOI: $10.3892 / \mathrm{mmr} .2017 .8296$

\begin{abstract}
Anoikis resistance is a crucial step in the process of tumor metastasis. This step determines whether the tumor cells will survive when they become detached from the extracellular matrix. However, the specific mechanism of tumor cells to bypass anoikis and become resistant remains to be elucidated. The present study aimed to determine the internal mechanism of bypassing anoikis through comparison of human osteosarcoma cell lines with human normal cell lines. High activating transcription factor 4 (ATF4) and myelocytomatosis oncogene (MYC) expression levels were observed in MG-63 and U-2 OS human osteosarcoma cell lines. It is possible that ATF4 and MYC contribute to tumor progression. Subsequently, the expression levels of ATF4 and MYC in HUVEC and CHON-001 human normal cell lines were upregulated and their adhesion abilities were reduced; whereas their ability to bypass anoikis increased significantly. Simultaneously, after we Following a knock-down of ATF4 and MYC expression levels in MG-63 and U-2 OS human osteosarcoma cell lines, their adhesion ability increased and their ability to bypassing anoikis was significantly reduced. Upregulation of MYC resulted in an upregulation of ATF4, and chromatin immunoprecipitation and luciferase reporter gene technology demonstrated that MYC binds to the promoter of ATF4. These findings suggest that ATF4 regulated by MYC might contribute to resistance to anoikis in human osteosarcoma cells.
\end{abstract}

Correspondence to: Dr Zhenchao Yuan, Department of Bone and Soft Tissue Neurosurgery, Affiliated Tumor Hospital of Guangxi Medical University, 71 He Di Road, Nanning, Guangxi 530021, P.R. China

E-mail: grzz5302321@126.com

${ }^{*}$ Contributed equally

Key words: osteosarcoma, anoikis, ATF4, MYC, transcriptional regulation

\section{Introduction}

Osteosarcoma is the most common bone tumor and accounts for $\sim 6 \%$ of all cancers in children and adolescents (1-3). Prior to the 1970s, the survival rate of surgical resection for osteosarcoma was 15-20\%. Presently, surgery combined with a neoadjuvant chemotherapy regimen increases the survival rate of osteosarcoma to $\sim 80 \%$ (4). The 5-year disease-free survival rate in the patients with localized disease can reach $65-70 \%$. However, this means that there are patients, which relapse within five years (5). The majority of osteosarcomas are diagnosed when they are already a high-grade malignant tumor at diagnosis and prone to early metastasis. The most common sites of metastases are the lungs, which account for $\sim 90 \%$ (6). With improved understanding of osteosarcoma and its invasion and metastasis mechanisms have become the focus of current research.

Apoptosis, also termed programmed cell death, is encoded by genes, which regulate spontaneous cell death process and cellular growth, differentiation, developmental and pathological processes. Normal epithelial or endothelial cells have adhesion dependency. Their survival depends on intercellular signaling and signaling between cells and the matrix is termed anchor-dependent (7). Normal epithelial cells or solid tumor cells, which do not have the ability to shed into the bloodstream from the extracellular matrix (ECM) will trigger apoptosis. This type of apoptosis occurs is termed anoikis (8).

The activating transcription factor (ATF) family encompasses a large group of transcription factors containing the leucine zipper basic region. ATF4 is a member of the ATF family. The influence of various stress factors may increase ATF4 expression levels, such as hypoxicconditions $(9,10)$, amino acid deprivation, endoplasmic reticulum and oxidative stress (11), and regulation of growth factor heregulin. ATF4 is an important protective gene that allows cells to adapt to the aforementioned stress factors. It has been previously reported that, compared with normal tissue, primary tumors in human tissue have a very high ATF4 protein expression levels. ATF4 may promote tumor growth in xenograft models (12). Myelocytomatosis oncogene (MYC) is the product of oncogene c-Myc. c-Myc is the first v-myc homolog to be confirmed to have a transformation effect in the MC29 avian retroviruses (13). MYC is widely expressed, particularly 
in cells and tissues that proliferate rapidly. Previous studies have determined that the function of MYC is associated with cell proliferation, differentiation and tumorigenesis (14-16).

A previous study reported that ATF4 may prevent anoikis and promote metastasis in human fibrosarcoma cells (17). The present study investigated the function of ATF4 and MYC in osteosarcoma cell lines. It was determined that ATF4 and MYC were highly expressed in the human osteosarcoma cell lines 143B and ZOS. Suspension culture of original adherent cells led to an increase in the expression levels of ATF4 and MYC. Additionally, overexpression of ATF4 and MYC in normal human cell lines was induced, where ATF4 and MYC usually have low expression levels. The present study determined that upregulation of ATF4 and MYC may significantly reduce the adherent ability of cells and aid them in bypassing anoikis. Conversely, a knockdown of ATF4 and MYC in human osteosarcoma cell lines may significantly increase the adherent ability of cells and increase the rate of anoikis. In these processes, MYC acted as a transcription factor regulating the expression of ATF4. Chromatin immunoprecipitation (ChIP) and luciferase reporter assays confirmed that MYC was able to bind to the promoter of ATF4 to regulate anoikis resistance.

\section{Materials and methods}

Cell culture. HUVEC, CHON-001, MG-63, U-2 OS and $293 \mathrm{~T}$ cell lines were obtained from American Type Culture Collection (Manassas, VA, USA). HUVECs were maintained in EGM-2 medium supplemented with $5 \mathrm{ng} / \mathrm{ml} \mathrm{rh}$ vascular endothelial growth factor, $5 \mathrm{ng} / \mathrm{ml} \mathrm{rh}$ EGF, $5 \mathrm{ng} / \mathrm{ml} \mathrm{rh}$ fibroblast growth factor basic, $15 \mathrm{ng} / \mathrm{ml} \mathrm{rh}$ IGF-1, $10 \mathrm{mM}$ L-glutamine, $0.75 \mathrm{U} / \mathrm{ml}$ heparin sulfate, $1 \mathrm{ng} / \mathrm{ml}$ hydrocortisone hemisuccinate, $2 \%$ fetal bovine serum (FBS) and $50 \mu \mathrm{g} / \mathrm{ml}$ ascorbic acid (Lonza, Inc., Allendale, NJ, USA). CHON-001 cells were maintained in Dulbecco's modified Eagle's medium supplemented with $0.1 \mathrm{mg} / \mathrm{ml} \mathrm{G}-418$ and 10\% FBS (Gibco; Thermo Fisher Scientific, Inc., Waltham, MA, USA). MG-63 cells were maintained in Eagle's minimum essential medium (Gibco; Thermo Fisher Scientific, Inc.) supplemented with $0.1 \mathrm{mg} / \mathrm{ml} \mathrm{G}-418$ and $10 \%$ FBS. U-2 OS cells were maintained in McCoy's 5A medium (Gibco; Thermo Fisher Scientific, Inc.) supplemented with $0.1 \mathrm{mg} / \mathrm{ml} \mathrm{G}-418$ and $10 \%$ FBS. 293T cells were maintained in Dulbecco's modified Eagle's medium (Gibco; Thermo Fisher Scientific, Inc.) supplemented with $10 \%$ FBS. Cells were cultured in a $37^{\circ} \mathrm{C}$ incubator with humidified atmosphere of $5 \% \mathrm{CO}_{2}$.

Induction of anoikis. To induce detachment from the ECM, four cell lines were cultured in a culture dish with a low attachment surface (cat no. 3473; Corning Incorporated, Corning, NY, USA). Suspension culture was performed for $24 \mathrm{~h}$. The mRNA and protein expression level changes of ATF4 and MYC were subsequently quantified.

RNA isolation from cells and reverse transcription-polymerase chain reaction $(R T-P C R)$ analysis. Total RNA was extracted from the cells using TRIzol (Invitrogen; Thermo Fisher Scientific, Inc.) and was reverse transcribed into cDNA using RevertAid First Strand cDNA Synthesis kit (Thermo
Fisher Scientific, Inc.). Taq polymerase (Invitrogen; Thermo Fisher Scientific, Inc.) was used for the PCR. The following thermocycling conditions were used: $94^{\circ} \mathrm{C} 5 \mathrm{~min} ; 32$ cycles of $94^{\circ} \mathrm{C} 30 \mathrm{sec}, 55^{\circ} \mathrm{C} 30 \mathrm{sec}, 72^{\circ} \mathrm{C} 1 \mathrm{~min} ; 72^{\circ} \mathrm{C} 10 \mathrm{~min}$. All experiments were performed in triplicate. Sequences of the primers for ATF4 were forward (F), 5'-TAGAGAAGTCCC GCCTCATAA-3' and reverse (R), 5'-TTCACTGCCCAGCTC TAAAC-3'; and MYC F, 5'-TTCTCTCCGTCCTCGGATT-3' and R, 5'-TGCGTAGTTGTGCTGATGT-3'.

Protein extraction from cells and western blot analysis. Total RNA was extracted from cells using Laemmli sample buffer with 5\% 2-mercaptoethanol (both from Bio-Rad Laboratories, Inc., Hercules, CA, USA). The Compat-Able ${ }^{\mathrm{TM}}$ BCA protein assay kit (cat no. 23229; Thermo Fisher Scientific, Inc.) was used to perform protein quantitation. A total of $40 \mu \mathrm{g}$ protein per lane was separated by $10 \%$ SDS-PAGE and transferred to polyvinylidene fluoride membranes. Blocking of the membranes was performed using $5 \%$ non-fat milk or $3 \%$ bovine serum albumin (cat no. A500023-0100; Sangon Biotech Co., Ltd., Shanghai, China). Primary antibodies used were ATF4 (cat no. ab50546; 1:5,000) and MYC (cat no. ab32072; 1:5,000) (both from Abcam, Cambridge, UK). GAPDH (cat no. G9545; 1:5,000; Sigma-Aldrich; EMD Millipore, Billerica, MA, USA) was used as normalization control. Primary antibodies were incubated with the membrane for $8 \mathrm{~h}$ at $4^{\circ} \mathrm{C}$. The membranes were washed three times with Tris-buffered saline with $0.2 \%$ Tween-20 and incubated with goat anti-mouse (cat no. ab6789; 1:2,000) and goat anti-rabbit secondary antibodies (cat no. ab6721; 1:2,000) (both from Abcam) labeled with horseradish peroxidase $>1 \mathrm{~h}$ at room temperature, and signals were visualized using film exposure. All experiments were performed in triplicate.

Construction of overexpression and knockdown vectors. The coding sequence of ATF4 and MYC was cloned into pcDNA3.1 vector (plasmid \#70219; Addgene, Inc., Cambridge, MA, USA) to overexpress them in cell lines. The following short hairpin (sh)RNAs were used to specifically target ATF4 and MYC and were cloned into pLKO.1 (plasmid \#10878; Addgene, Inc.) vector to induce knockdown of their expression in cell lines. All experiments were performed in triplicate. shRNA-1 targeted ATF4 sequence, GCCTAGGTCTCTTAGATGATT; shRNA-2 targeted ATF4 sequence, CCACTCCAGATCATT CCTTTA; shRNA-1 targeted MYC sequence, CAGTTGAAA CACAAACTTGAA; shRNA-2 targeted MYC sequence, CCT GAGACAGATCAGCAACAA.

Adhesion assay. Six-well plates were coated with $10 \mathrm{mg} / \mathrm{ml}$ fibronectin (cat no. F2006; Sigma-Aldrich; Merck KGaA) overnight at $4^{\circ} \mathrm{C}$. The cell concentration was adjusted to $2 \times 10^{5}$ cells $/ \mathrm{ml}$, and $500 \mu \mathrm{l}$ was added to each well. The cells were incubated for $30 \mathrm{~min}$ at $37^{\circ} \mathrm{C}$, and unattached cells were discarded. Adhered cells were stained with crystal violet $(0.005 \%)$ for $10 \mathrm{~min}$, and the cells were photographed with a microscope under a 40x objective. The number of adherent cells was averaged from 10 fields.

Cell Death Detection ELISA. The level of anoikis was detected using a Cell Death Detection ELISA ${ }^{\text {PLuS }}$ kit (cat 
no. 11920685001; Roche Diagnostics, Basel, Switzerland), according to the manufacturer's protocol.

ChIP. ChIP assay was performed using an Imprint ${ }^{\circledR}$ Chromatin Immunopreciptitation kit (cat no. CHP1; Sigma-Aldrich; Merck KGaA). Cells were crosslinked with $1 \%$ formaldehyde and terminated with $2.5 \mathrm{mM}$ glycine. Briefly, the scraped cells were lysed in PBS + sodium thiosulfate using a sonicator, and the lysates were divided into three. One part was used as a positive control and received no treatment. The second was used as negative control and was incubated with IgG (cat no. ab150077; $5 \mu \mathrm{g} / \mathrm{ml}$; Abcam) and $100 \mu 1$ Protein G PLUS-Agarose. The third lysate was incubated with MYC-specific antibody $(5 \mu \mathrm{g} / \mathrm{ml})$ and Protein A/G PLUS-Agarose at $4^{\circ} \mathrm{C}$ overnight. Lysates were incubated with $10 \mu \mathrm{g}$ RNaseA (cat no. R6148; Sigma-Aldrich; Merck KGaA) for $30 \mathrm{~min}$ at $37^{\circ} \mathrm{C}$, and $80 \mu \mathrm{g}$ proteinase K (cat no. P2308; Sigma-Aldrich; Merck KGaA) for $3 \mathrm{~h}$ at $37^{\circ} \mathrm{C}$, to remove the RNA and protein. DNA was subsequently extracted with using phenol-chloroform. The degree of enrichment was detected using quantitative PCR with the following thermocycling parameters: $94^{\circ} \mathrm{C}, 5 \mathrm{~min} ; 40$ cycles of $94^{\circ} \mathrm{C}$ for $30 \mathrm{sec}, 55^{\circ} \mathrm{C}$ for $15 \mathrm{sec}, 72^{\circ} \mathrm{C}$ for $1 \mathrm{~min} ; 72^{\circ} \mathrm{C}$ for $10 \mathrm{~min}$. R1 of the control was used for normalization. Power SYBR $^{\circledR}$ Green PCR Master Mix (cat no. 4368708; Applied Biosystems; Thermo Fisher Scientific, Inc.) was used to perform qPCR. The quantification performed using the $\Delta \Delta \mathrm{Cq}$ method (18) and all experiments were performed in triplicate. The ChIP primers used to measure enrichment are detailed in Table I.

Luciferase reporter gene technology. MG-63 (2x10 $)$ and U-2 OS $\left(2 \times 10^{5}\right)$ cells were plated into a 24 -well plate and transfected with $2 \mathrm{ng}$ of cytomegalovirus (CMV)-Renilla (Promega Corporation, Madison, WI, USA) and $10 \mathrm{ng}$ of ATF4-dependent luciferase reporter constructs using Lipofectamine 2000 transfection reagent (Thermo Fisher Scientific, Inc.). Cells were harvested $36 \mathrm{~h}$ after transfection. Luciferase activity was quantified using the Dual-Luciferase reporter system (Promega Corporation). All experiments were performed in triplicate.

Statistical analysis. Statistical analyses were performed using SPSS version 17.0 (SPSS, Inc., Chicago, IL, USA). Statistical differences were determined using a Student's t-test or one-way analysis of variance followed by Tukey's honest significant difference test. $\mathrm{P}<0.05$ was considered to indicate a statistically significant difference.

\section{Results}

ATF4 and MYC are highly expressed in human osteosarcoma cell lines. ATF4 is a transcription factor associated with several types of cancer progression, such as breast cancer, lung cancer and melanoma (19-21). It has been determined that ATF4 expression is induced by stress stimulation, such as endoplasmic reticulum stress, anoxia/hypoxia and amino acid deprivation. MYC is also a transcription factor that has been identified as a proto-oncogene. MYC is expressed with relatively high probability in tumor cells to regulate proliferation, apoptosis, differentiation and cell cycle
Table I. ChIP primers.

\begin{tabular}{ll}
\hline Primer & \multicolumn{1}{c}{ Sequence $\left(5^{\prime}-3^{\prime}\right)$} \\
\hline R1 forward & tgtccgtgtgtcatcttggtt \\
R1 reverse & atactgaatgggcagatga \\
R2 forward & acataggcaattgctttgagat \\
R2 reverse & aaagttactcatctttcccca \\
R3 forward & agtaggtgtttacctttaca \\
R3 reverse & gcaggcaagtgactagaaggc \\
R4 forward & gcaaggatactcatcagtagt \\
R4 reverse & cgcatccaaagaatcctggct \\
R5 forward & gctggtcctgaggccactaa \\
R5 reverse & tctcgggtcgctgctagtcctca \\
R6 forward & gatcgggaaagcgtagtcgggt \\
R6 reverse & ttcgtggggcaacagccaagac \\
R7 forward & gagaaaatggatttgaagga \\
R7 reverse & aagagatcacaagtgtcatcc
\end{tabular}

$\mathrm{R}$, region.

progression. However, the interaction between these two proteins remains to be elucidated. The present study determined that ATF4 and MYC mRNA and protein expression levels were high in MG-63 and U-2 OS human osteosarcoma cell lines (Fig. 1A and B). Therefore, ATF4 and MYC may have an important role in the progression of osteosarcoma. The present study focused on the association between these two genes and anoikis. It was determined that the expression levels of ATF4 and MYC markedly increased following the suspension culture (Fig. 1C and D). Therefore, ATF4 and MYC may have an important function in anoikis resistance observed in human osteosarcoma cells.

Increased expression of ATF4 and MYC allows normal human cell lines to escape anoikis. Dey et al (17) have determined that ATF4 prevented anoikis in human fibrosarcoma cell lines. Disease-associated fibronectin matrix fragments trigger anoikis of human primary ligament cells through suppression of c-myc (22). The present study investigated whether increased expression levels of ATF4 and MYC allowed cells to bypass anoikis in human normal cell lines. Initially, a lentiviral packaging plasmid and target plasmid overexpressing ATF4 or MYC were transfected into 293T cells. Subsequently, lentiviral vectors were collected and used to infect HUVEC and CHON-001 human normal cell lines. Subsequently ATF4 and MYC protein expression levels were detected (Fig. 2A and B).

HUVEC and CHON-001 cells overexpressing ATF4 and MYC were used to conduct adhesion assay. This assay was used to detect the ability of cells to escape the ECM by observing the number of cells adhered on the dish coated with fibronectin. The present study determined that the adhesion ability of HUVEC and CHON-001 cells overexpressing ATF4 or MYC decreased significantly (Fig. 2C and D). This suggested that overexpression of ATF4 and MYC may promote cells to become detached and initiate metastasis. 


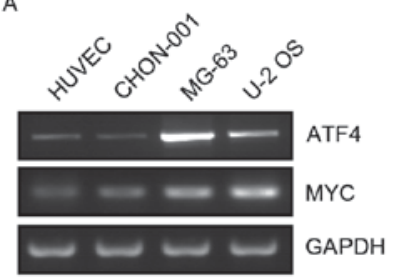

C

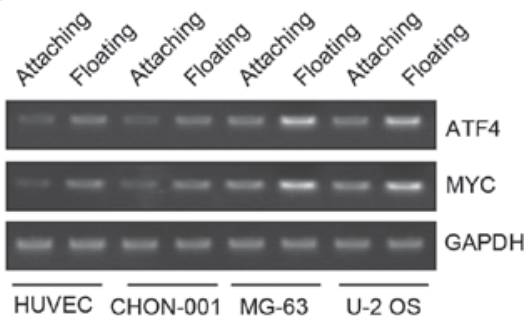

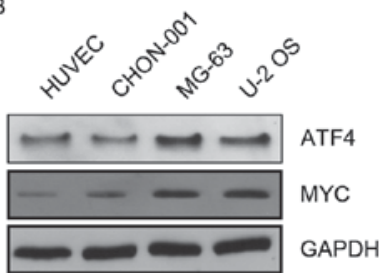

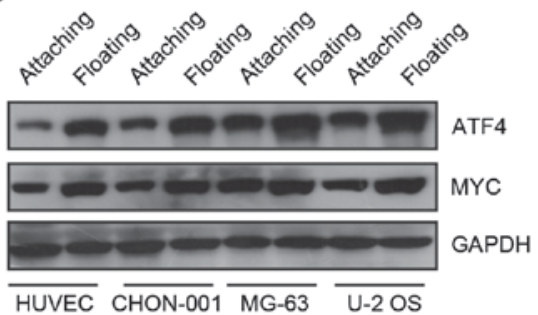

Figure 1. ATF4 and MYC were expressed in human osteosarcoma cell lines. (A and B) Comparable levels of ATF4 and MYC in mRNA and protein levels in HUVEC, CHON-001, MG-63 and U-2 OS cell lines. (C and D) Comparable levels of ATF4 and MYC in mRNA and protein levels in attaching and floating cell lines. ATF4, activating transcription factor 4; MYC, myelocytomatosis oncogene.

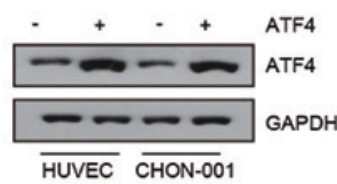

c
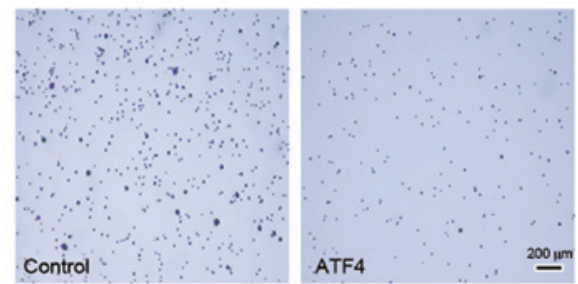

D
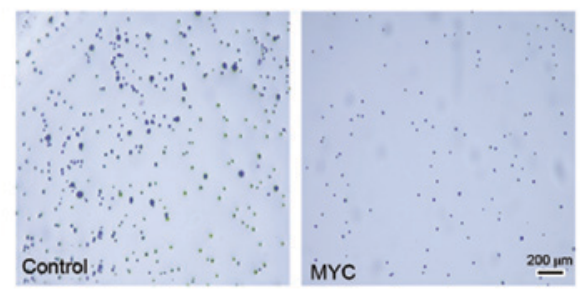

E

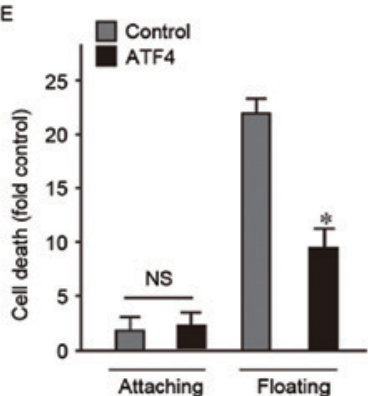

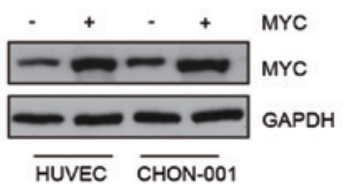

$\overline{\text { HUVEC }} \overline{\mathrm{CHON}-001}$
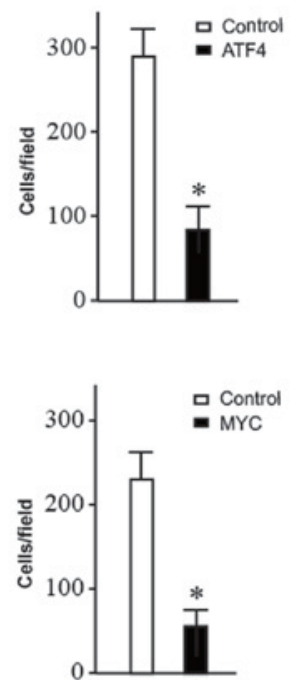

F

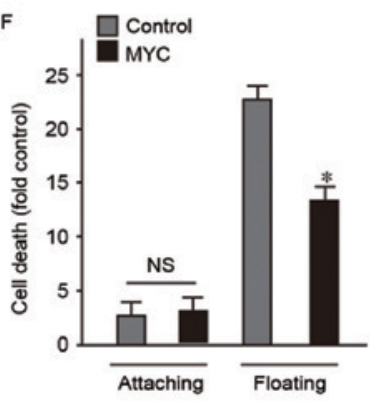

Figure 2. Upregulation of ATF4 and MYC contributed to bypass anoikis in human normal cell lines. (A and B) ATF4 and MYC were overexpressed in HUVEC and CHON-001 cell lines. (C and D) Overexpression of ATF4 and MYC reduced the adhesion ability of HUVEC. Scale bar, 200 $\mu$ m. (E and F) Overexpression of ATF4 and MYC contributed HUVEC bypass anoikis. ${ }^{*} \mathrm{P}<0.05$. NS, no significance; ATF4, activating transcription factor 4; MYC, myelocytomatosis oncogene. 


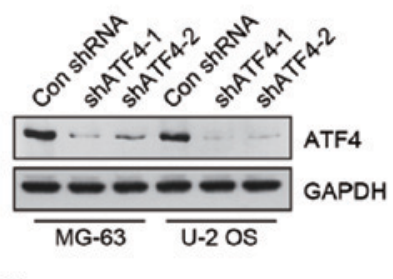

$c$
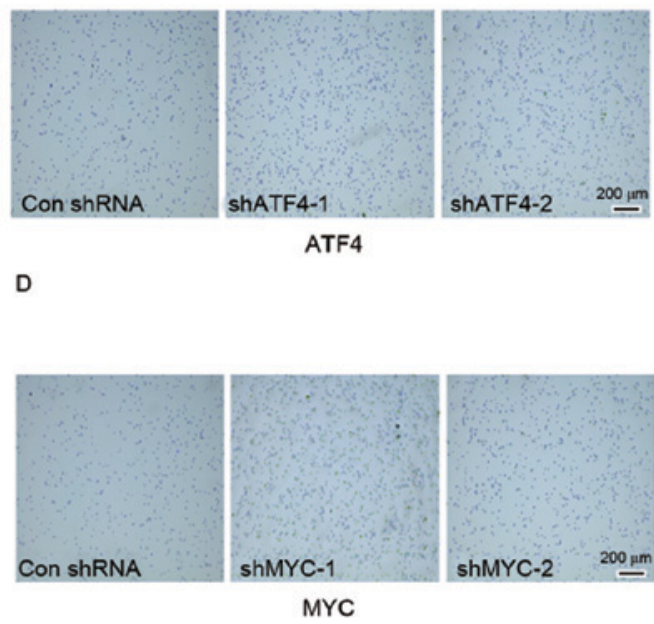

E

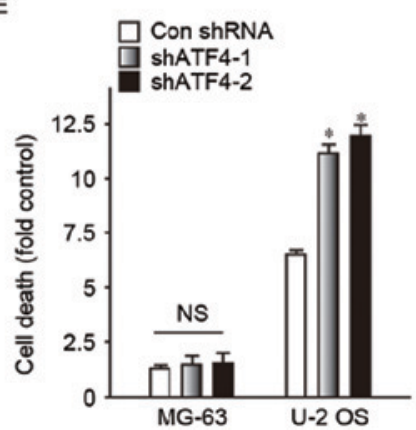

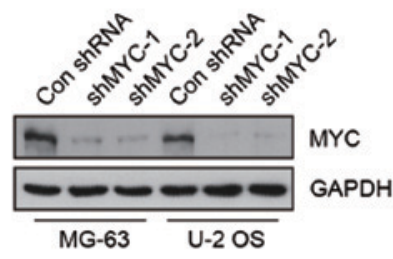
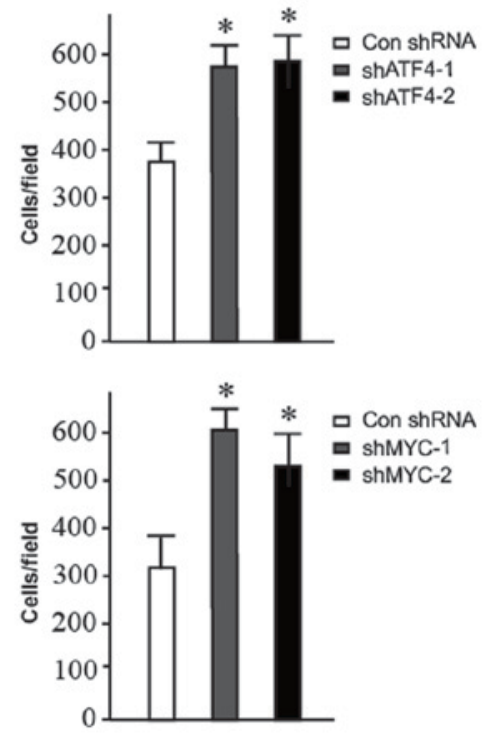

$\mathbf{F}$

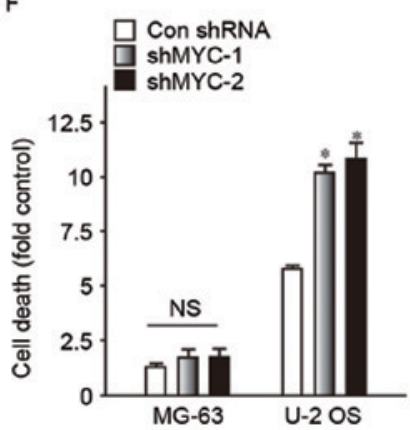

Figure 3. Knockdown of ATF4 and MYC in human osteosarcoma cells promoted anoikis. (A and B) ATF4 and MYC was knocked-down in MG-63 and U-2 OS cell lines. (C and D) Knockdown of ATF4 and MYC improved the adhesion ability of MG-63 cells. Scale bar, $200 \mu$ m. (E and F) Knockdown of ATF4 and MYC increased the ratio of anoikis in MG-63 cells. "P<0.05 vs. control. NS, no significance; sh, short hairpin; ATF4, activating transcription factor 4; MYC, myelocytomatosis oncogene.

As ATF4 and MYC may facilitate detachment of cells from the ECM they may also aid cells in bypassing anoikis. The Cell Death Detection ELISA ${ }^{\text {PLUS }}$ kit to quantify cell death following suspension culture for $24 \mathrm{~h}$. The mortality of HUVEC and CHON-001 floating cells overexpressing ATF4 and MYC was significantly reduced compared with the control (Fig. 2E and F). These findings indicated that ATF4 and MYC contributed to the cells' ability to become detached and bypass anoikis. Therefore, ATF4 and MYC may have a crucial role in the carcinogenesis process.

Knockdown of ATF4 and MYC in human osteosarcoma cells promotes anoikis. In order to confirm that ATF4 and MYC have a role in improving cell viability following detachment from ECM during carcinogenesis, shRNA was used to reduce ATF4 and MYC expression levels in MG-63 and U-2 OS human osteosarcoma cells. Two clones were designed for two different target sequences of ATF4 and MYC respectively, vector-shATF4-1, vector-shATF4-2, vector-shMYC-1 and vector-shMYC-2. The ATF4 and MYC protein expression levels in the transfected cell lines were determined (Fig. 3A and B). Subsequently, adhesion assay and cell death detection ELISA were performed to confirm that MG-63 and U-2 OS cells, following knock-down of ATF4 and MYC had reduced detachment abilities and unable to bypassing anoikis. Cells preferred to adhere to the bottom of the dish (Fig. 3C and D) in the knock-down of ATF4 and MYC groups. Following a suspension culture for $24 \mathrm{~h}$, cells in the knock-down groups had significantly increased mortality compared with the control group (Fig. 3E and F). Therefore, it was evident that the downregulation of ATF4 and MYC expression may increase cell adhesion and induce apoptosis due to loss of cell-matrix 


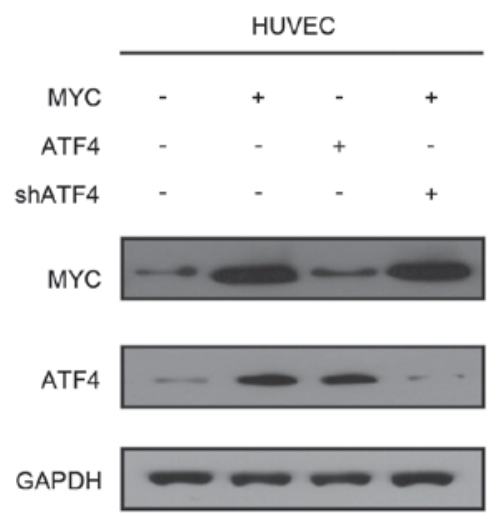

C

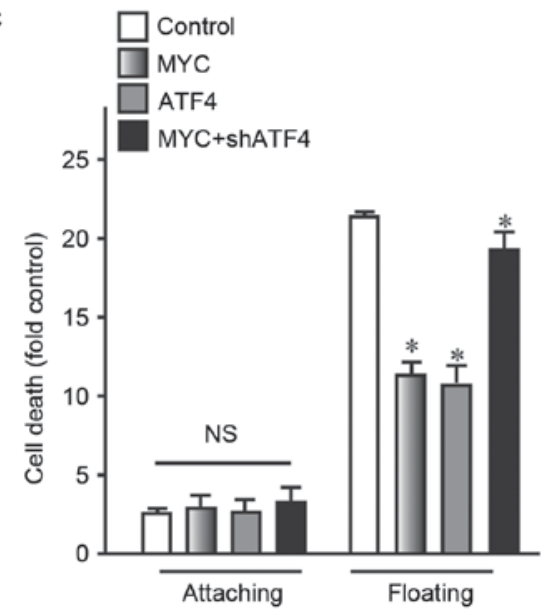

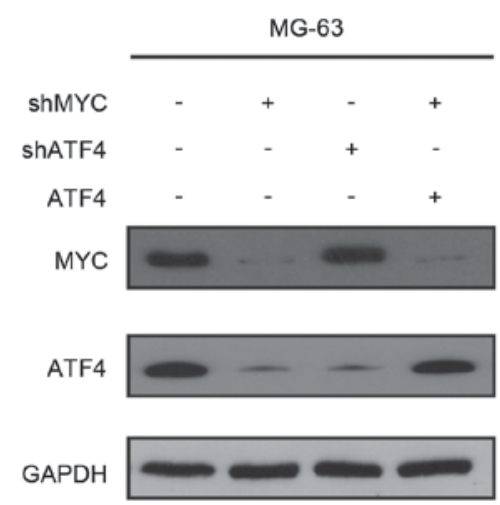

$\mathrm{D}$

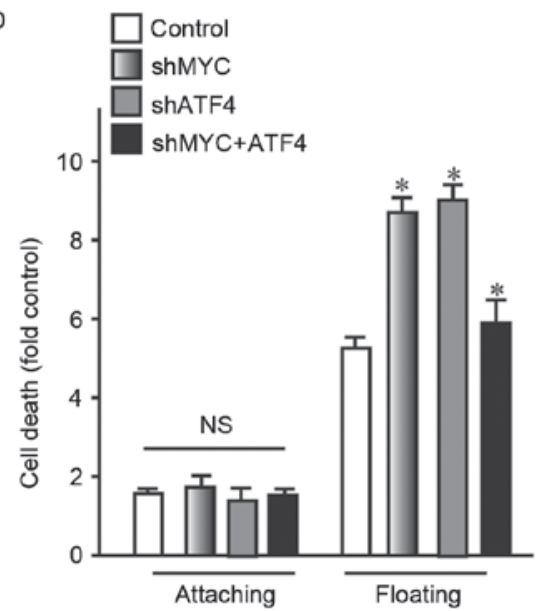

Figure 4. Expression levels of ATF4 change following alteration of MYC expression. HUVECs and MG-63 cells were transfected with (A) MYC, ATF4 or MYC+shATF4-1 and (B) shMYC, shATF4 or shMYC+ATF4. (C and D) Cell death in the treatment groups was assessed after $24 \mathrm{~h}$ under attached or floating conditions. " $\mathrm{P}<0.05$ vs. control. NS, no significance; sh, short hairpin; ATF4, activating transcription factor 4; MYC, myelocytomatosis oncogene.

interactions. This confirmed that ATF4 and MYC contribute to the process of cancer metastasis, particularly in the aspect of bypassing anoikis.

Expression of ATF4 changes following alteration of MYC expression. The present study aimed to determine how ATF4 and MYC function together in the carcinogenesis process. Therefore, the association between ATF4 and MYC has been further investigated.

To detect this association, ATF4 and MYC were overexpressed in human normal cell lines HUVEC and CHON-001. Subsequently, their expression levels were detected. The findings revealed that overexpressing MYC may lead to significantly increased expression levels of ATF4. However, overexpression of ATF4 did not lead to alteration of MYC expression levels in HUVEC cells (Fig. 4A). Similarly, knockdown of MYC did not alter the expression of ATF4 (Fig. 4B). As two the two different vectors used for the aforementioned ATF4 and MYC knock-down experiments exhibited a similar effect, shATF4-1 and shMYC-1 were selected for this experiment. These findings demonstrated that MYC may act as an upstream regulator of ATF4 expression.

The present study also examined whether anoikis resistance ability of cells was restored following partial upregulation of ATF4 in cells with upregulation of MYC expression. Following
$24 \mathrm{~h}$ suspension culture, cells with restored ATF4 exhibited reduced cell death, compared with the control (Fig. 4C and D). These findings confirmed that ATF4 and MYC were associated with anoikis. Additionally, MYC may act as a transcription factor regulating the expression of ATF4 and ATF4 may activate downstream signaling pathways associated with anoikis.

MYC binds to the promoter of ATF4 to regulate its expression. The present study has demonstrated that MYC may act as an upstream regulatory factor of ATF4 expression, further affecting cell sensitivity to anoikis-mediated cell death. ChIP was used to further investigate the specific mechanism of the underlying molecular process where MYC regulates ATF4, in order to determine where MYC may bind onto the promoter region of ATF4. Encyclopedia of DNA Elements at UCSC database (genome.ucsc.edu/ENCODE/) predicted that there was the possible binding site for MYC in the promoter region of ATF4. Subsequently, 7 regions were selected (R1 R7) near the transcription starting site. They were $\sim 1 \mathrm{~kb}$ apart from one another (Fig. 5A). HUVEC and CHON-001 cells overexpressing MYC or transfected with a control plasmid were used to conduct ChIP. DNA regions associated with MYC would be pulled down together with via MYC affinity with the MYC monoclonal antibody. R5 was determined to be the enrichment region of MYC in the ATF4 promoter region (Fig. 5B and C). 
A

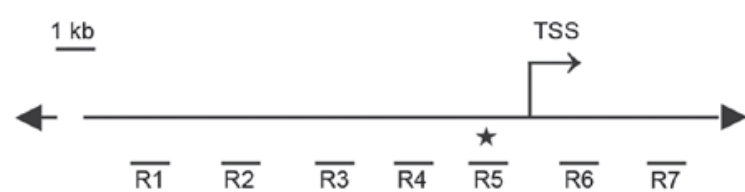

B

HUVEC
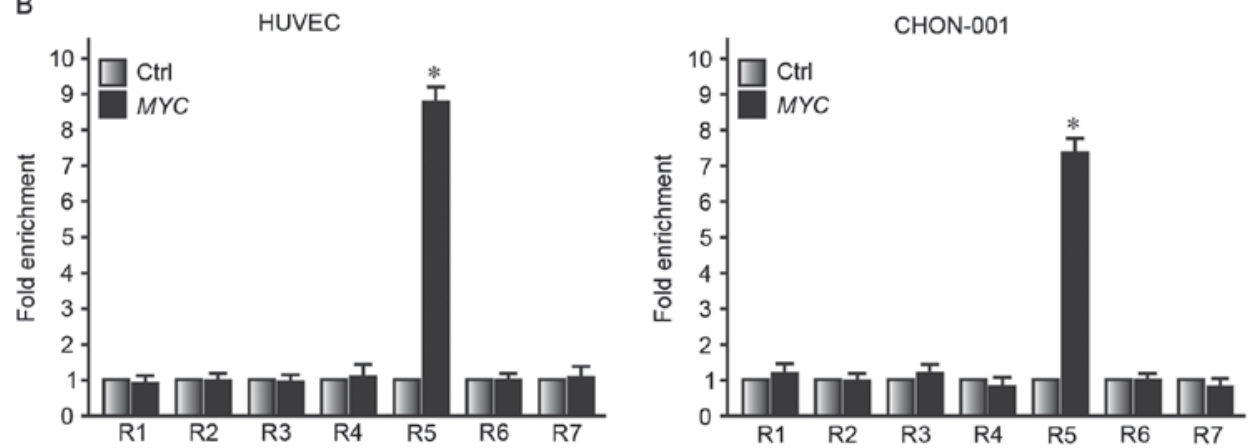

C
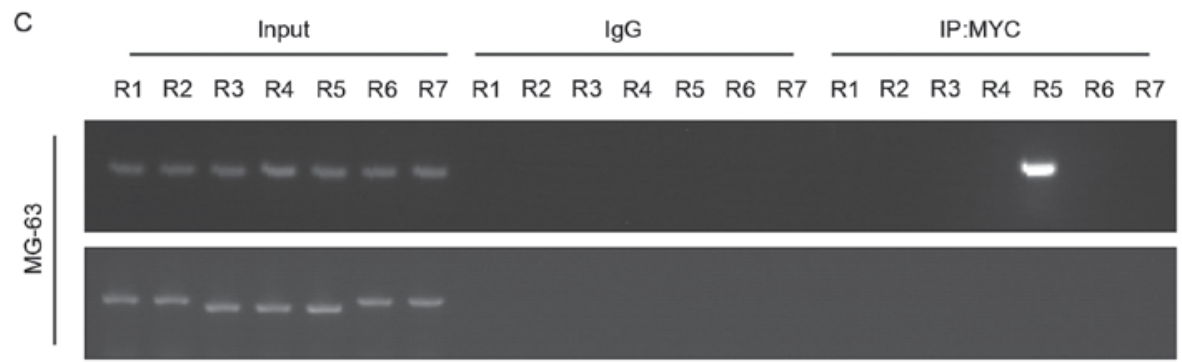

Overexpression

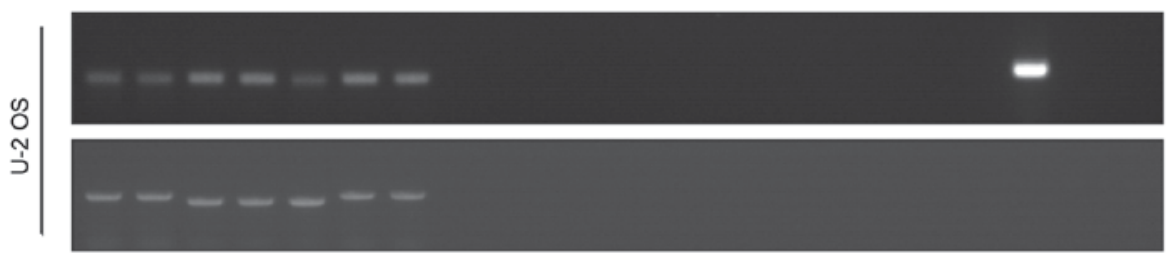

MYC

Control vector

MYC

Control vector

D
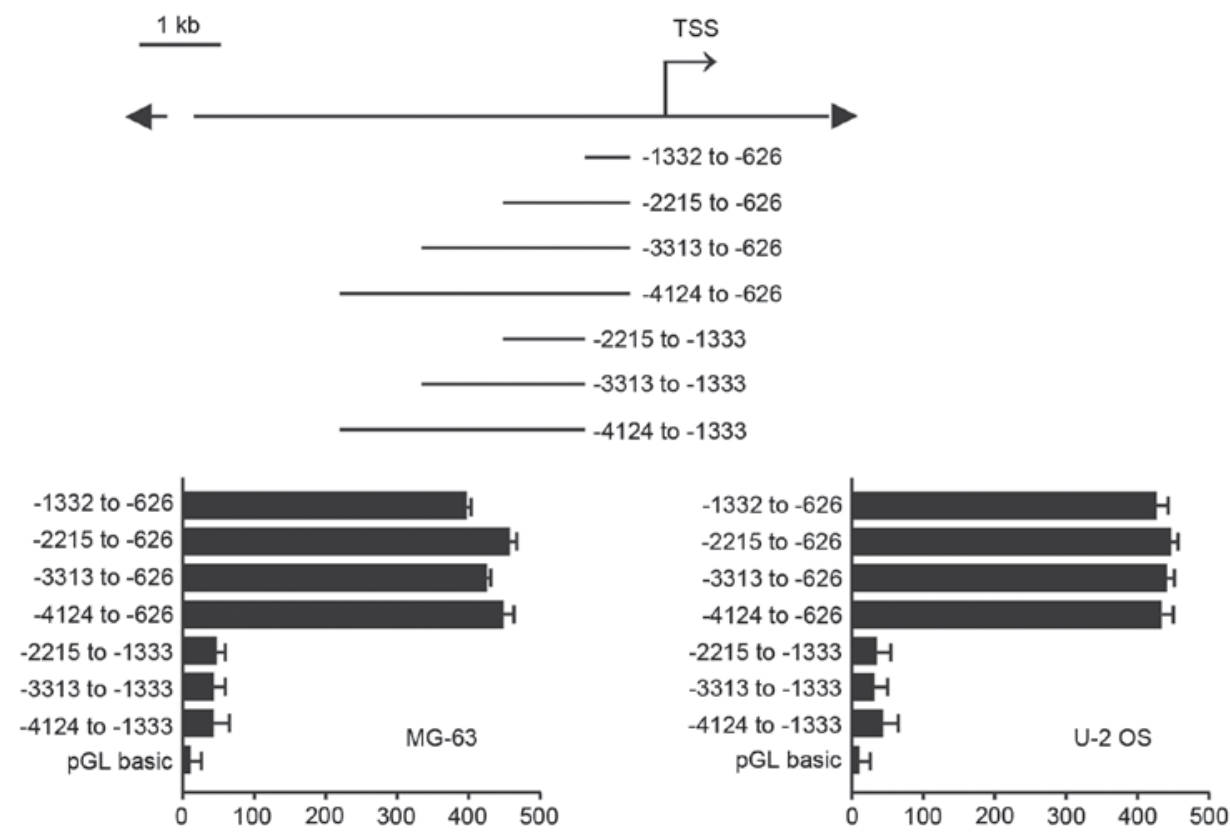

Figure 5. MYC binds to the promoter of ATF4 to regulate its expression. (A) A total of 7 regions were designed of ChIP. (B and C) ChIP analysis demonstrated the association of MYC in transfected HUVEC and CHON-001 cells in region 5. (D) Luciferase reporter assays were performed with the -1,332 to -626 segment-containing region where MYC regulates ATF4. ATF4, activating transcription factor 4; MYC, myelocytomatosis oncogene; ChIP, chromatin immunoprecipitation; TSS, transcription start site.. 
Luciferase reporter was transfected into MG-63 and U-2 OS, which express MYC at high levels. It was determined that high luciferase reporter signal was detected at the $-1,332$ to -626 bp fragment (Fig. 5D). Therefore, the findings of the present study demonstrated that MYC may bind to the ATF4 promoter region and regulate its expression level, thus altering anoikis resistance in human osteosarcoma cells.

\section{Discussion}

Osteosarcoma is a primary malignant bone tumor, which originates in mesenchymal cells. Typical osteosarcoma clinical is rare and its incidence is $\sim 300 / 1,000$, accounting for $0.2 \%$ of malignant tumors and $15 \%$ of primary bone tumors (23). Prior to 1970 , the standard treatment for osteosarcoma was amputation. However, $80 \%$ of patients have been determined to have micrometastasis at the time of diagnosis (24). In recent years, limb salvage treatment of osteosarcoma has gradually replaced amputation, with the development of chemical treatments, surgical techniques and improved treatment methods of bone reconstruction. A previous study reported that $>80 \%$ of patients preferred limb salvage surgery (25). Nevertheless, the metastasis mechanism of osteosarcoma remains fully elucidated.

Anoikis as a specific type of programmed cell death, which is important role for development, organism homeostasis, disease and tumor metastasis. It has been previously reported that suppression of anoikis was closely associated with metastasis and survival of tumor cells (26). Previous studies determined that expression of cancer-associated genes, such as Ras, may prevent anoikis in normal epithelial cells (27-29). Subsequent studies have reported that inhibition of anoikis was associated with the malignant degree of breast, colon and lung cancer (30-32). The Fas cell surface death receptor and its adapter molecule Fas-associated via death domain have been determined to be involved in anoikis (33). Integrins have also been identified to be closely associated with anoikis (34). Cell adhesion and anoikis may be mediated by integrins depending on the cell type and anoikis model selected (35). Additionally, adhesion and detachment between cell and matrix alter Bcl-2 expression levels. Therefore, adjusting the balance of anti-apoptotic and apoptosis signals determines cell survival or death by anoikis (36). A previous study determined that after cells detach from the ECM, FAK does not bind to PI3K and inhibits its activity, eventually, promotes cells anoikis (37).

The present study determined that MYC may bind to the promoter region of ATF4 and regulated its expression to adjust anoikis resistance in human osteosarcoma cells. A previous study determined that ATF4-dependent induction of heme oxygenase 1 prevents anoikis and promotes metastasis in a human fibrosarcoma cell line (17). The effect of MYC on anoikis has also been previously reported as mitochondrial DNA depletion prevented anoikis through upregulation of PI3K, which led to the phosphorylation of downstream substrates Myc (38). The present study determined that ATF4 has the same function in anoikis resistance different cell lines. However, the current study also identified a completely different mechanism where MYC acts as a transcription factor, not a downstream gene, which regulated ATF4 to trigger anoikis resistance.
To the best of our knowledge, this study is the first to confirm that ATF4 and MYC promote anoikis resistance in human osteosarcoma. Additionally, it was revealed that MYC affects anoikis resistance through regulation of ATF4. ATF4 and MYC are likely to become potential targets for future cancer therapy. However, the underlying mechanism of ATF4-induced anoikis resistance remains to be elucidated. Furthermore, the role of ATF4 and MYC in anoikis resistance requires verification in other types of cancer.

\section{Acknowledgements}

The present study was supported by Science Computing and Intelligent Information Processing of GuangXi Higher Education Key Laboratory (grant no. GXSCIIP201502).

\section{References}

1. Damron TA, Ward WG and Stewart A: Osteosarcoma, chondrosarcoma, and Ewing's sarcoma: National cancer data base report. Clin Orthop Relat Res 459: 40-47, 2007.

2. Rainusso N, Wang LL and Yustein JT: The adolescent and young adult with cancer: State of the art-bone tumors. Curr Oncol Rep 15: 296-307, 2013.

3. Sweetnam R: Osteosarcoma. Br J Hosp Med 28: 112, 116-121, 1982.

4. Sun L, Li Y, Li H, Zhang J, Li B and Ye Z: Analysis of chemotherapy dosage and dosage intensity and survival outcomes of high-grade osteosarcoma patients younger than 40 years. Clin Ther 36: 567-578, 2014

5. Hayden JB and Hoang BH: Osteosarcoma: Basic science and clinical implications. Orthop Clin North Am 37: 1-7, 2006.

6. Krishnan K, Khanna C and Helman LJ: The biology of metastases in pediatric sarcomas. Cancer J 11: 306-313, 2005.

7. Cheng TL, Symons M and Jou TS: Regulation of anoikis by Cdc42 and Rac1. Exp Cell Res 295: 497-511, 2004.

8. Frisch SM and Francis H: Disruption of epithelial cell-matrix interactions induces apoptosis. J Cell Biol 124: 619-626, 1994.

9. Ameri K, Lewis CE, Raida M, Sowter H, Hai T and Harris AL: Anoxic induction of ATF-4 through HIF-1-independent pathways of protein stabilization in human cancer cells. Blood 103: 1876-1882, 2004.

10. Blais JD, Filipenko V, Bi M, Harding HP, Ron D, Koumenis C, Wouters BG and Bell JC: Activating transcription factor 4 is translationally regulated by hypoxic stress. Mol Cell Biol 24: 7469-7482, 2004.

11. Harding HP, Zhang Y, Zeng H, Novoa I, Lu PD, Calfon M, Sadri N, Yun C, Popko B, Paules R, et al: An integrated stress response regulates amino acid metabolism and resistance to oxidative stress. Mol Cell 11: 619-633, 2003.

12. Bi M, Naczki C, Koritzinsky M, Fels D, Blais J, Hu N, Harding H, Novoa I, Varia M, Raleigh J, et al: ER stress-regulated translation increases tolerance to extreme hypoxia and promotes tumor growth. Embo J 24: 3470-3481, 2005.

13. Vennstrom B, Sheiness D, Zabielski J and Bishop JM: Isolation and characterization of c-myc, a cellular homolog of the oncogene (v-myc) of avian myelocytomatosis virus strain 29. J Virol 42: 773-779, 1982.

14. Schuhmacher M and Eick D: Dose-dependent regulation of target gene expression and cell proliferation by c-Myc levels. Transcription 4: 192-197, 2013.

15. Wall M, Poortinga G, Hannan KM, Pearson RB, Hannan RD and McArthur GA: Translational control of c-MYC by rapamycin promotes terminal myeloid differentiation. Blood 112: 2305-2317, 2008.

16. O'Donnell KA, Yu D, Zeller KI, Kim JW, Racke F, Thomas-Tikhonenko A and Dang CV: Activation of transferrin receptor 1 by c-Myc enhances cellular proliferation and tumorigenesis. Mol Cell Biol 26: 2373-2386, 2006.

17. Dey S, Sayers CM, Verginadis II, Lehman SL, Cheng Y, Cerniglia GJ, Tuttle SW, Feldman MD, Zhang PJ, Fuchs SY, et al: ATF4-dependent induction of heme oxygenase 1 prevents anoikis and promotes metastasis. J Clin Invest 125: 2592-2608, 2015. 
18. Livak KJ and Schmittgen TD: Analysis of relative gene expression data using real-time quantitative PCR and the 2-(Delta Delta C(T)) method. Methods 25: 402-408, 2001

19. Ferguson J, Smith M, Zudaire I, Wellbrock C and Arozarena I: Glucose availability controls ATF4-mediated MITF suppression to drive melanoma cell growth. Oncotarget 8: 32946-32959, 2017.

20. Hsu HY, Lin TY, Lu MK, Leng PJ, Tsao SM and Wu YC: Fucoidan induces Toll-like receptor 4-regulated reactive oxygen species and promotes endoplasmic reticulum stress-mediated apoptosis in lung cancer. Sci Rep 7: 44990, 2017.

21. Yuan X, Kho D, Xu J, Gajan A, Wu K and Wu GS: ONC201 activates ER stress to inhibit the growth of triple-negative breast cancer cells. Oncotarget 8: 21626-21638, 2017.

22. Dai R, Iwama A, Wang S and Kapila YL: Disease-associated fibronectin matrix fragments trigger anoikis of human primary ligament cells: p53 and c-myc are suppressed. Apoptosis 10: 503-512, 2005.

23. Bishop MW, Janeway KA and Gorlick R: Future directions in the treatment of osteosarcoma. Curr Opin Pediatr 28: 26-33, 2016.

24. Rosen G, Tan C, Sanmaneechai A, Beattie EJ Jr, Marcove R and Murphy ML: The rationale for multiple drug chemotherapy in the treatment of osteogenic sarcoma. Cancer 35 (3 Suppl): S936-S945, 1975.

25. Jaffe N: Osteosarcoma: Review of the past, impact on the future. The American experience. Cancer Treat Res 152: 239-262, 2009.

26. Frisch SM and Screaton RA: Anoikis mechanisms. Curr Opin Cell Biol 13: 555-562, 2001.

27. Debnath J: p66 (Shc) and Ras: Controlling anoikis from the inside-out. Oncogene 29: 5556-5558, 2010.

28. Halim H, Luanpitpong S and Chanvorachote P: Acquisition of anoikis resistance up-regulates caveolin-1 expression in human non-small cell lung cancer cells. Anticancer Res 32: 1649-1658, 2012.

29. Hu Y, Chen H, Duan C, Liu D, Qian L, Yang Z, Guo L, Song L, $\mathrm{Yu} \mathrm{M}, \mathrm{Hu} \mathrm{M}$, et al: Deficiency of Erbin induces resistance of cervical cancer cells to anoikis in a STAT3-dependent manner. Oncogenesis 2: e52, 2013.
30. Mahauad-Fernandez WD and Okeoma CM: Cysteine-linked dimerization of BST-2 confers anoikis resistance to breast cancer cells by negating proapoptotic activities to promote tumor cell survival and growth. Cell Death Dis 8: e2687, 2017.

31. Chieng CK and Say YH: Cellular prion protein contributes to LS 174T colon cancer cell carcinogenesis by increasing invasiveness and resistance against doxorubicin-induced apoptosis. Tumour Biol 36: 8107-8120, 2015

32. Yao X, Pham T, Temple B, Gray S, Cannon C, Chen R, Abdel-Mageed AB and Biliran H: The anoikis effector Bit1 inhibits EMT through attenuation of TLE1-mediated repression of E-cadherin in lung cancer cells. PloS One 11: e0163228, 2016.

33. Aoudjit F and Vuori K: Matrix attachment regulates Fas-induced apoptosis in endothelial cells: A role for c-flip and implications for anoikis. J Cell Biol 152: 633-643, 2001.

34. Frisch SM and Ruoslahti E: Integrins and anoikis. Curr Opin Cell Biol 9: 701-706, 1997.

35. Grossmann J: Molecular mechanisms of 'detachment-induced apoptosis-anoikis'. Apoptosis 7: 247-260, 2002.

36. Matter ML and Ruoslahti E: A signaling pathway from the alpha5beta1 and alpha (v)beta3 integrins that elevates bcl-2 transcription. J Biol Chem 276: 27757-27763, 2001.

37. Monteiro HP, Silva EF and Stern A: Nitric oxide: A potential inducer of adhesion-related apoptosis-anoikis. Nitric Oxide 10: $1-10,2004$.

38. Moro L, Arbini AA, Yao JL, di Sant'Agnese PA, Marra E and Greco M: Mitochondrial DNA depletion in prostate epithelial cells promotes anoikis resistance and invasion through activation of PI3K/Akt2. Cell Death Differ 16: 571-583, 2009. International (CC BY-NC-ND 4.0) License. 